NBER WORKING PAPER SERIES

A TESTABLE THEORY OF IMPERFECT PERCEPTION

\author{
Andrew Caplin \\ Daniel Martin \\ Working Paper 17163 \\ http://www.nber.org/papers/w17163
}

NATIONAL BUREAU OF ECONOMIC RESEARCH
1050 Massachusetts Avenue
Cambridge, MA 02138
June 2011

We thank Nabil Al-Najjar, David Cesarini, Mark Dean, Jeff Ely, Sen Geng, Paul Glimcher, Natalia Shestakova, Jonathan Weinstein, and seminar participants at Northwestern University and University of Southern California for valuable comments. The views expressed herein are those of the authors and do not necessarily reflect the views of the National Bureau of Economic Research.

NBER working papers are circulated for discussion and comment purposes. They have not been peerreviewed or been subject to the review by the NBER Board of Directors that accompanies official NBER publications.

(C) 2011 by Andrew Caplin and Daniel Martin. All rights reserved. Short sections of text, not to exceed two paragraphs, may be quoted without explicit permission provided that full credit, including $\odot$ notice, is given to the source. 
A Testable Theory of Imperfect Perception

Andrew Caplin and Daniel Martin

NBER Working Paper No. 17163

June 2011

JEL No. D01,D03

\begin{abstract}
$\underline{\text { ABSTRACT }}$
We introduce a rational choice theory that allows for many forms of imperfect perception, including failures of memory, selective attention, and adherence to simplifying rules of thumb. Despite its generality, the theory has strong, simple, and intuitive implications for standard choice data and for more enriched choice data. The central assumption is rational expectations: decision makers understand the relationship between their perceptions, however limited they may be, and the (stochastic) consequences of their available choices. Our theory separately identifies two distinct "framing" effects: standard effects involving the layout of the prizes (e.g. order in a list) and novel effects relating to the information content of the environment (e.g. how likely is the first in the list to be the best). Simple experimental tests both affirm the basic model and confirm the existence of information-based framing effects.
\end{abstract}

\author{
Andrew Caplin \\ Department of Economics \\ New York University \\ 19 W. 4th Street, 6th Floor \\ New York, NY 10012 \\ and NBER \\ andrew.caplin@nyu.edu \\ Daniel Martin \\ Department of Economics \\ New York University \\ 19 W. 4th Street, 6th Floor \\ New York, NY 10012 \\ daniel.martin@nyu.edu
}




\title{
A Testable Theory of Imperfect Perception*
}

\author{
Andrew Caplin ${ }^{\dagger}$ and Daniel Martin ${ }^{\ddagger}$
}

June 15, 2011

\begin{abstract}
We introduce a rational choice theory that allows for many forms of imperfect perception, including failures of memory, selective attention, and adherence to simplifying rules of thumb. Despite its generality, the theory has strong, simple, and intuitive implications for standard choice data and for more enriched choice data. The central assumption is rational expectations: decision makers understand the relationship between their perceptions, however limited they may be, and the (stochastic) consequences of their available choices. Our theory separately identifies two distinct "framing" effects: standard effects involving the layout of the prizes (e.g. order in a list) and novel effects relating to the information content of the environment (e.g. how likely is the first in the list to be the best). Simple experimental tests both affirm the basic model and confirm the existence of information-based framing effects.
\end{abstract}

Key Words: Stochastic Choice, Bounded Rationality, Imperfect Perception, Rational Expectations, Framing Effects, Mistakes

\section{Introduction}

From Weber [1834] on, psychologists have explored the gap between subjective perceptions and external reality (Glimcher [2010]). In recent years, economists have also worked to incorporate this

\footnotetext{
${ }^{*}$ We thank Nabil Al-Najjar, David Cesarini, Mark Dean, Jeff Ely, Sen Geng, Paul Glimcher, Natalia Shestakova, Jonathan Weinstein, and seminar participants at Northwestern University and University of Southern California for valuable comments.

${ }^{\dagger}$ Center for Experimental Social Science and Department of Economics, New York University and National Bureau of Economic Research.

${ }^{\ddagger}$ Center for Experimental Social Science and Department of Economics, New York University.
} 
gap into the theory of rational decision making. To date, progress has typically involved making various highly specific (and mutually incompatible) assumptions concerning perceptual limitations, including bounds on memory, rigid mental accounts, incomplete and selective attention, incomplete search, rule of thumb decision making protocols, algorithmic approximations, etc. (e.g. Thaler [1985], Mullainathan [2002], Wilson [2002], Sims [2003], Manzini and Mariotti [2007], Rabin and Weizsacker [2009], Masatlioglu and Nakajima [2009], Gennaioli and Shleifer [2010], Gottlieb [2010], Schwartzstein [2010], Ergin and Sarver [2010], Caplin and Dean [2011], Compte and Postlewaite [2011], and Gabaix [2011]).

An open question is how to introduce less circumscribed forms of imperfect perception into decision theory. What makes this challenging is that, without some form of theoretical discipline, rational choice theory becomes vacuous. Any pattern of behavior can be rationalized by some distorted perception of reality. This is related to the difficulties in identifying "mistaken" decisions from choice data, and the dangers of so doing without strong theoretical guidance. ${ }^{1}$

We introduce a model of rational decision making that allows for a wide array of perceptual limitations and mistakes, yet has strong and intuitive testable implications. The main substantive assumption is rational expectations. In the "rational expectations perception-based representations" (RE-PREPs) that we study, decision makers (DMs) fully understand the relationship between their subjective perceptions, however limited they may be, and the (stochastic) consequences of available choices. The restrictions that our theory imposes on choice data are simple to understand: choices must be "unimprovable". The precise form of the improvements that are ruled out depend on the data available for model testing. We produce separate results for "framed" stochastic choice data and for standard stochastic choice data.

When the frame is observable, our theory separately identifies two distinct framing effects: standard effects involving the layout of the prizes (Rubinstein and Salant [2006], Salant and Rubinstein [2008], Bernheim and Rangel [2008], Reutskaja, Nagel, Camerer, and Rangel [2011]) and novel effects relating to the information content of the environment (e.g. the extent to which the first in the list turns out to be the best). In this setting, we show that existence of a RE-PREP representation is equivalent to a "No Improving Action Switches" (NIAS) condition: there must be a utility function such that no action can be switched to an alternative action in a manner that raises utility. This restriction corresponds to the non-emptiness of the feasible set for a data-defined

\footnotetext{
${ }^{1}$ See Koszegi and Rabin [2008], Bernheim and Rangel [2008], and Gul and Pesendorfer [2008].
} 
linear program.

We present experimental tests that affirm the basic model and confirm the importance of information-based framing effects. While subjects make substantial choice mistakes in all treatments, we find that the NIAS inequalities are always satisfied. Further, both of the framing effects allowed by our model are present in the experimental data. Our experiment provides proof-ofconcept that our information-based framing effect can be experimentally identified, and that such identification can further understanding of the perceptual process. It also highlights the symbiotic relationship between decision theory and experiments that generate non-standard choice data (see also Caplin [2008], Caplin and Dean [2011], and Caplin, Dean, and Martin [forthcoming]).

In addition to its implications for framed stochastic choice data, our theory places simple and intuitive restrictions on standard stochastic choice data. These restrictions are weaker than those imposed by stochastic utility models (e.g. Block and Marschak [1960], Luce [1959], McFadden [1973], Falmange [1978], and Gul and Pesendorfer [2006]). By way of example, our theory covers cases in which perceptual similarities between a very good prize and a very bad prize result in the selection of a moderately good but distinctive prize when all three are available (e.g. Debreu [1960] and Natenzon [2011]). ${ }^{2}$

In section 2 we introduce our formal model and define RE-PREP representations. In sections 3 and 4 we provide the observable restrictions associated with the model in cases in which the decision making environment can be fully observed: the NIAS conditions. In section 5 we consider the various framing effects that our model encompasses. In section 6 we characterize the restrictions our model places on standard stochastic choice data. A theoretically-inspired experiment for understanding the sources and nature of imperfect perception is presented in section 7 . Concluding remarks are in section 8 .

\section{Decision Making and Perceptions}

We study decisions in which the DM may not fully understand all objects of choice. This may be because there are many available options, because these items are complex, or because the DM is

\footnotetext{
${ }^{2}$ In this sense, our results relate to the question posed more than fifty years ago by Block and Marschak [1960] concerning how to distinguish stochastic choices with decision making errors from stochastic choices based on stochastic utility.
} 
not able or willing to expend the effort to become thoroughly familiar with the available options. This is the world envisaged in models of bounded rationality at least since the pioneering work of Simon [1955].

\subsection{Prizes, Actions, and Layouts}

We assume that there is an "ideal observer" (IO) who understands fully the choice environment and who is distinct from the DM whose choice behavior is being modeled. The IO knows not only the key ingredients of the decision making environment that the DM is facing, but also the choices that are made and the prizes received.

A crucial feature of the model is that we distinguish the act of choice from the receipt of a prize. It is through separate observation of actions and of their consequences that the IO in our model is able to interpret some of the DM's choices as based on misperception.

In formal terms, there is a finite prize set $X$ with generic element $x_{n}$, for $n \in\{1, \ldots, N\}$ : this fixes the physical presentation of each option once and for all, including its packaging, etc. There is a separate set $Y$ of action choices that is also finite and has at least the same cardinality as the prize set,

$$
|Y|=M \geq|X|=N \geq 2
$$

Each $y \in Y$ is to be physically interpreted (e.g. a location on a screen or a line in a list). A layout is an onto function $f: Y \rightarrow X$, with $\mathcal{F}$ the set of all such layouts,

$$
\mathcal{F}=\{f: Y \rightarrow X \mid f \text { is onto }\} .
$$

It is the layout that connects actions with consequences. The assumption that layouts are onto ensures that all prizes in $X$ are in fact available to the DM. In certain cases we restrict the function to be one-to-one (see section 6). However, allowing for the more general case enables the model to cover such phenomena as "needle in a haystack" choice sets, in which one good prize is obscured by the availability of several equivalent bad prizes. It is just such choice sets that underlie the experiment of section 7 .

Our model draws strongly from the psychological tradition. In particular, our formulation of the act of choice as separate from reception of a prize was inspired by simple choice experiments 
of the drift-diffusion form (see Ratcliff [1978], Ratcliff and McKoon [2008], Shadlen and Newsome $[2001])$.

\subsection{Choice Environment}

If a given action choice $y \in Y$ always produced the same prize, one would expect the DM to learn this, making the question of misperception moot. However, when there is doubt about how actions get translated into prizes, there is room for misperception. We study a DM making many independent choices in a stable environment identified with a specific layout-generating mechanism (a probability measure over layouts) $\mu \in \Delta(\mathcal{F})$. We define $\mathcal{F}(\mu) \subset \mathcal{F}$ as the support of $\mu$. We refer to the triple $(X, Y, \mu)$ as the choice environment.

In principle, the measure $\mu$ provides the subject a statistical sense of what tends to happen when a given action is selected. A DM's choices may be influenced both by this information and by information derived from attending to the action-prize association in a given layout.

\subsection{Ideal Data}

Given $\mu \in \Delta(\mathcal{F})$, an ideal data set (IDS) $P$ identifies the probability distribution over action choices as it depends on the layout,

$$
P: \mathcal{F} \longrightarrow \Delta(Y)
$$

This entire function is assumed to be known to the IO and provides the data that a theory of choice must explain. ${ }^{3}$ It is the quadruple $(X, Y, \mu, P)$ that fully identifies the objects upon which our theory of perception is built. We call such a quadruple an ideal choice environment (ICE).

Our model allows the structure of observed choices to be dependent on the layout. For example, we allow for situations in which the first item in a list is most likely to be the best, which may induce default behavior of selecting this option. In such cases, the stochastic structure of choice may be impacted by which object is put first in the list. For that reason, a direct test of our theory requires that the set of action choices $Y$ is identifiable to the IO, and that the choice environment $\mu$ is also understood. Such knowledge is most naturally derived in an experiment, and plays a key

\footnotetext{
${ }^{3}$ The domain of this data does not allow the order of the experimental runs to be recorded, hence we will not consider explanatory hypotheses that involve learning about the structure of the experiment.
} 
role in the experimental design of section 7 . In section 6 we consider cases in which the layout is unobservable, and the only available data relates to stochastic demand for prizes.

\subsection{Subjective States and the Perception Function}

The IO takes as given an ICE $(X, Y, \mu, P)$ and looks to structure perceptual theories of choice based on three elements: a subjective state space $S$, a perception function $\rho$ that maps possible layouts into simple lotteries over the subjective state space, and a choice function $C$ that maps possible subjective states into action choices.

The set $S$ specifies in the IO's model all permissible subjective mental states of the DM. In general, $S$ captures all decision relevant information the IO hypothesizes DMs to extract from the choice environment and the specific layout in front of them. More specifically, $S$ may encode characteristics or facets of the available goods, the state of a finite automaton, etc. Note that this set need not be an accurate representation of the DM's state of mind, rather it is the manner in which the state is modeled by the IO in seeking to understand observed behavioral patterns.

In the current context, the IO is presumed to set $S=\Delta(X)^{M}$, the space of ordered lists of $M$ subjective prize lotteries. Let $s_{m n}$ be the probability that action choice $y_{m}$ results in prize $x_{n}$, so that,

$$
S=\left\{s \in \mathbb{R}_{+}^{M N} \mid \sum_{n=1}^{N} s_{m n}=1 \text { for all } m \in\{1, \ldots, M\}\right\} .
$$

The reason the IO so limits the subjective state space is that the desired characterization of behavior is based on expected utility theory, for which lotteries over prizes are the appropriate objects of choice.

The perception function maps possible layouts $f \in \mathcal{F}(\mu)$ into $\Delta(S)$, the probability distributions over $S$ with finite support,

$$
\rho: \mathcal{F}(\mu) \rightarrow \Delta(S)
$$

Given finite support, the set of lottery states that are perceived as possible in the given experiment,

$$
S(\rho)=\cup_{f \in \mathcal{F}(\mu)}\left\{s \in S \mid \rho^{f}(s)>0\right\},
$$

is finite. It is this set that serves as the domain of the choice function,

$$
C: S(\rho) \longrightarrow Y
$$




\subsection{RE-PREPs}

Since the space $S$ is fixed from this point forward, one can specify a perceptual model by the two elements $\rho$ and $C$. For these to provide a possible explanation (perception-based representation or PREP) of the observed data requires that their composition generates the IDS. We are interested only in PREPs in which the DM can be modeled as an expected utility maximizer with rational expectations. We require strictness in the utility comparison of some pair of acts in some state of mind to prevent the conditions from being trivially satisfied by a utility function in which all utilities are identical.

Definition 1 ( $\rho, C, U)$ form a rational expectations perception-based representation (REPREP) of $(X, Y, \mu, P)$ if they satisfy:

1. Data Matching: $P^{f}(y)=\rho^{f}\left(C^{-1}(y)\right)$ for all $f \in F$ and $y \in Y$.

2. Rational Expectations: For all $m \in\{1, \ldots, M\}, n \in\{1, \ldots, N\}$, and $s \in S(\rho)$,

$$
s_{m n}=\frac{\sum_{\left\{f \in \mathcal{F}(\mu) \mid f\left(y_{m}\right)=x_{n}\right\}} \mu(f) \rho^{f}(s)}{\sum_{\{f \in \mathcal{F}(\mu)\}} \mu(f) \rho^{f}(s)} .
$$

3. Optimality: If $C(s)=y_{m}$, then,

$$
\sum_{n=1}^{N} s_{m n} U\left(x_{n}\right) \geq \sum_{n=1}^{N} s_{k n} U\left(x_{n}\right) \text { all } k \in\{1, \ldots, M\},
$$

with the inequality being strict for some pair $k, m$ with $k, m \in\{1, \ldots, M\}$.

Note that if there is only one action ever taken, so that there exists $y \in Y$ with $C(s)=y$ for all $s \in S$, then it is trivial to find a RE-PREP by setting $y$ as the unique utility maximizer. Hence we will consider only cases in which there are at least two distinct actions taken with strictly positive probability.

\subsection{Rational Expectations and Experimentation}

The most distinctive aspect of the RE-PREP is Rational Expectations, which when combined with Data Matching, implies that the DM is aware of the consequences of all choices in each state 
of mind. What this means is that in a RE-PREP, the statistical association between subjective perceptions, choices, and prizes has been internalized by the DM. Implicitly, this is based on the idea that this is a familiar environment and that DMs have learned through a process of trial and error what the results are when any given choices are made in any particular state of mind. Having thus experimented, each selects an optimal such action, and the consequences are then at least acceptable, in the sense that they do not perceive there to be any action choice that is superior. The full statement of this intuitive description of the axioms is formalized in our representation theorem.

As is often the case, the assumption of rational expectations is easiest to justify as the end result of an unmodeled and unobserved process of experimentation. However, in the actual definition, a specific deterministic action is taken in each state of mind, and there is no experimentation whatsoever. The exact process of experimentation or decision making tremble that would produce rational expectations and thereby rationalize the strong informational assumption that the REPREP imbeds is not modeled.

\section{Restrictions on Ideal Data: the $2 \times 2 \times 2$ Case}

Our goal is to identify conditions on the IDS equivalent to existence of a RE-PREP. Before introducing the general characterization theorem in the next section, we provide in this section a thorough analysis of the $2 \times 2 \times 2$ case, with two prizes $X=\left\{x_{1}, x_{2}\right\}$; two actions $Y=\left\{y_{1}, y_{2}\right\}$; and two possible layouts $\mathcal{F}=\{g, h\}$, in which actions yield different prizes,

$$
\begin{aligned}
& g\left(y_{1}\right)=h\left(y_{2}\right)=x_{1} ; \\
& g\left(y_{2}\right)=h\left(y_{1}\right)=x_{2} .
\end{aligned}
$$

In this setting, one parameter (the probability of layout $g$ ) identifies the layout-generating mechanism,

$$
\mu^{g} \in[0,1]
$$

while two parameters $p_{1}^{g}, p_{1}^{h} \in[0,1]$ identify the IDS,

$$
\begin{aligned}
& \left(P^{g}\left(y_{1}\right), P^{g}\left(y_{2}\right)\right)=\left(p_{1}^{g}, 1-p_{1}^{g}\right) \\
& \left(P^{h}\left(y_{1}\right), P^{h}\left(y_{2}\right)\right)=\left(p_{1}^{h}, 1-p_{1}^{h}\right) .
\end{aligned}
$$


To avoid triviality, we assume that both choices are made with strictly positive probability.

Throughout this section, we will look for RE-PREPs using two perceptual states,

$$
S=\left\{s^{1}, s^{2}\right\}
$$

By definition,

$$
s_{11}^{i}, s_{21}^{i} \in[0,1], i=1,2,
$$

denote the probability in subjective lottery state $s^{i}$ that actions $y_{1}, y_{2}$ respectively will yield prize $x_{1}$, with,

$$
s_{12}^{i}=1-s_{11}^{i}, s_{22}^{i}=1-s_{21}^{i},
$$

representing the corresponding probabilities for prize $x_{2}$. Given the assumption that both choices are observed, it must be that $C\left(s^{1}\right) \neq C\left(s^{2}\right)$. Hence there is no loss of generality in setting $C\left(s^{i}\right)=y_{i}$ for $i=1,2$. We show in the next section that allowance for more than two subjective states is unnecessary, so that the analysis of this section is more general than it appears.

\subsection{Decision Tree Representation}

Figures 1 and 2 illustrate the structure of this RE-PREP. Figure 1 is a representation of all states of the world as directly seen by the IO, with the top branch recording the stochastic structure of the layout, the second branch the stochastic choice of action that is observed with each layout, and the final vertical line representing the deterministic association of the prize with the action as it depends on the layout.

Figure 2 presents the elements of the theory of DM behavior that the IO hypothesizes to be responsible for DM choices. The main change from figure 1 is the addition of nodes corresponding to the two subjective states, $s^{1}$ and $s^{2}$. These nodes are placed after the layout and prior to the action stage. The subjective states are connected with dashed lines, which represent the information sets of the DM. Each node has a black edge that goes to the action that is adopted in the RE-PREP, action $y_{i}$ in state $s^{i}$, and also a grey edge corresponding to the untaken alternative action. Note that the branches of the tree that lead to the mental states retain the same probability labels as in figure $1, \rho^{g, h}\left(s^{1}\right)=p_{1}^{g, h}$ and $\rho^{g, h}\left(s^{2}\right)=p_{2}^{g, h}$, on the relevant branches of the tree. This reflects 


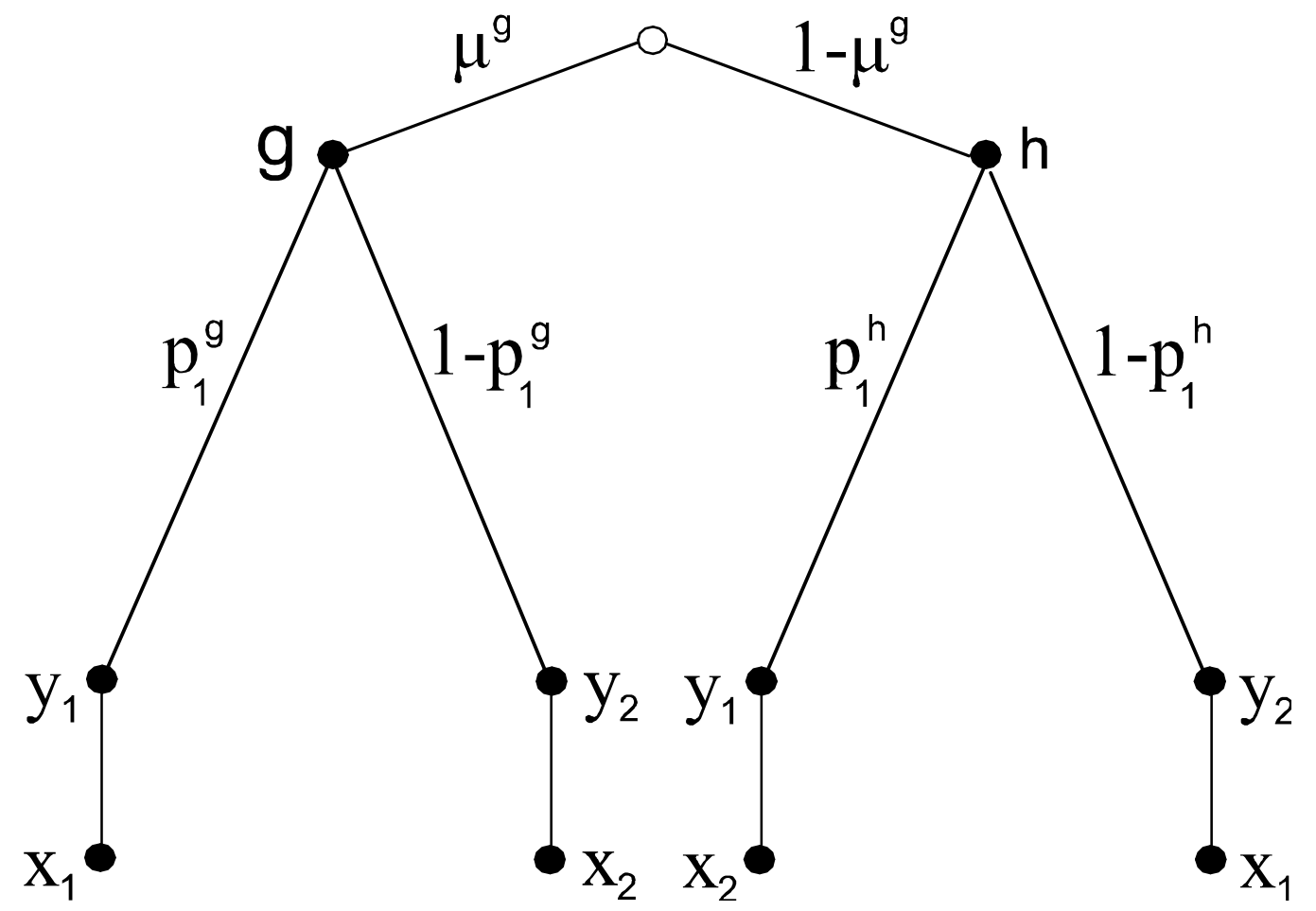

Figure 1: Decision tree as seen by "ideal observer" (IO)

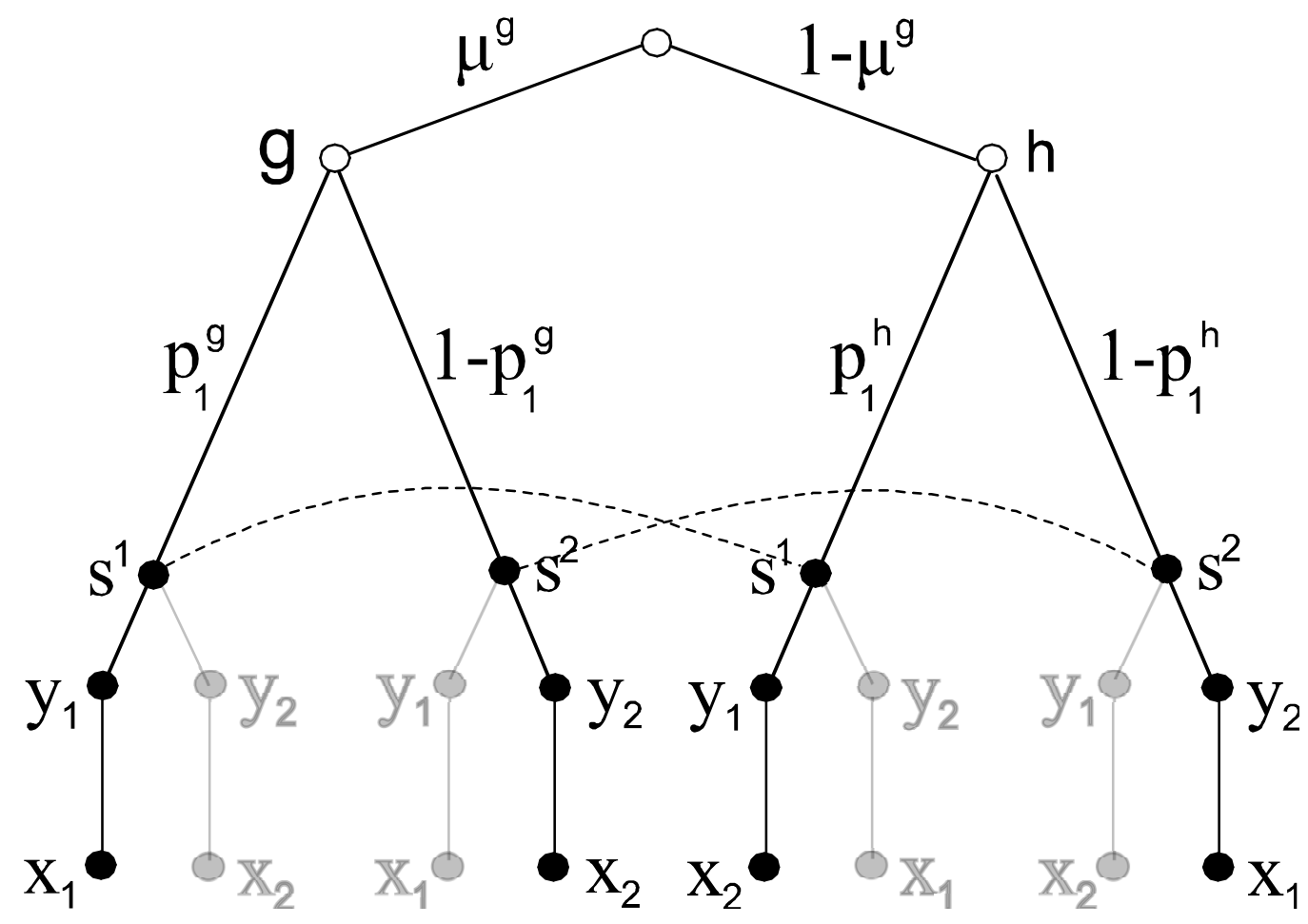

Figure 2: Decision tree for DM as hypothesized by IO 
application of the first property of a RE-PREP, Data Matching, which requires that,

$$
\begin{aligned}
& \rho^{g}\left\{C^{-1}\left(y_{1}\right)\right\}=\rho^{g}\left(s^{1}\right)=p_{1}^{g} \\
& \rho^{h}\left\{C^{-1}\left(y_{1}\right)\right\}=\rho^{h}\left(s^{1}\right)=p_{1}^{h} .
\end{aligned}
$$

The question of whether or not a RE-PREP exists hinges on whether or not the Rational Expectations condition and the Optimality condition can simultaneously be met.

\subsection{Existence: An Example}

Our first example involves $\mu^{g}=0.5, p_{1}^{g}=0.8$, and $p_{1}^{h}=0.2$. In this case, substitution for $\rho$ based on Data Matching reveals the Rational Expectations conditions to be:

$$
\begin{aligned}
& s_{11}^{1}=\frac{\mu^{g} p_{1}^{g}}{\mu^{g} p_{1}^{g}+\left(1-\mu^{g}\right) p_{1}^{h}}=0.8=\frac{\left(1-\mu^{g}\right)\left(1-p_{1}^{h}\right)}{\mu^{g}\left(1-p_{1}^{g}\right)+\left(1-\mu^{g}\right)\left(1-p_{1}^{h}\right)}=s_{21}^{2} ; \\
& s_{21}^{1}=\frac{\left(1-\mu^{g}\right) p_{1}^{h}}{\mu^{g} p_{1}^{g}+\left(1-\mu^{g}\right) p_{1}^{h}}=0.2=\frac{\mu^{g}\left(1-p_{1}^{g}\right)}{\mu^{g}\left(1-p_{1}^{g}\right)+\left(1-\mu^{g}\right)\left(1-p_{1}^{h}\right)}=s_{11}^{2} .
\end{aligned}
$$

The remaining lottery probabilities $s_{12}^{1}=s_{22}^{2}=0.2$ and $s_{22}^{1}=s_{12}^{2}=0.8$ are implied. With this, the conditions for Optimality of the chosen action are the same for each action:

$$
0.8 U\left(x_{1}\right)+0.2 U\left(x_{2}\right) \geq 0.2 U\left(x_{1}\right)+0.8 U\left(x_{2}\right) .
$$

To ensure (as required for a RE-PREP) that this inequality is strict, we set,

$$
U\left(x_{1}\right)>U\left(x_{2}\right)
$$

completing the specification of the RE-PREP representation.

\subsection{Non-Existence: An Example}

An example that has no RE-PREP involves $\mu^{g}=0.8$ and $p_{1}^{g}=p_{1}^{h}=0.5$. In this case, substitution for $\rho$ based on Data Matching reveals the Rational Expectations conditions to be:

$$
\begin{aligned}
& s_{11}^{1}=\frac{\mu^{g} p_{1}^{g}}{\mu^{g} p_{1}^{g}+\left(1-\mu^{g}\right) p_{1}^{h}}=0.8=\frac{\mu^{g}\left(1-p_{1}^{g}\right)}{\mu^{g}\left(1-p_{1}^{g}\right)+\left(1-\mu^{g}\right)\left(1-p_{1}^{h}\right)}=s_{11}^{2} ; \\
& s_{21}^{1}=\frac{\left(1-\mu^{g}\right) p_{1}^{h}}{\mu^{g} p_{1}^{g}+\left(1-\mu^{g}\right) p_{1}^{h}}=0.2=\frac{\left(1-\mu^{g}\right)\left(1-p_{1}^{h}\right)}{\mu^{g}\left(1-p_{1}^{g}\right)+\left(1-\mu^{g}\right)\left(1-p_{1}^{h}\right)}=s_{21}^{2}
\end{aligned}
$$


with remaining lottery probabilities $s_{12}^{1}=s_{12}^{2}=0.2$ and $s_{22}^{1}=s_{22}^{2}=0.8$ implied. With this, the conditions for Optimality depend on the state and are inverses of one another:

$$
\begin{aligned}
& 0.8 U\left(x_{1}\right)+0.2 U\left(x_{2}\right) \geq 0.2 U\left(x_{1}\right)+0.8 U\left(x_{2}\right) ; \\
& 0.2 U\left(x_{1}\right)+0.8 U\left(x_{2}\right) \geq 0.8 U\left(x_{1}\right)+0.2 U\left(x_{2}\right) .
\end{aligned}
$$

The only utility functions that solve this involve $U\left(x_{1}\right)=U\left(x_{2}\right)$, but in this case neither inequality holds strictly, contrary to the definition of a RE-PREP.

\subsection{Restrictions on the IDS}

We now identify necessary and sufficient conditions on the IDS for existence of a RE-PREP for general $p_{1}^{g}, p_{1}^{h}, \mu^{g} \in(0,1)$ in the two state world of figure 2 with $C\left(s^{i}\right)=y_{i}, i=1,2$. The Data Matching condition is as implied in the figure with $\rho^{g}\left(s^{i}\right)=p_{i}^{g}$ and $\rho^{h}\left(s^{i}\right)=p_{i}^{h}$ for $i=1,2$. Substitution for $\rho$ then reveals the Rational Expectations conditions to be:

$$
\begin{aligned}
s_{11}^{1} & =\frac{\mu^{g} p_{1}^{g}}{\mu^{g} p_{1}^{g}+\left(1-\mu^{g}\right) p_{1}^{h}}=s_{22}^{1} \\
s_{11}^{2} & =\frac{\mu^{g}\left(1-p_{1}^{g}\right)}{\mu^{g}\left(1-p_{1}^{g}\right)+\left(1-\mu^{g}\right)\left(1-p_{1}^{h}\right)}=s_{22}^{2} .
\end{aligned}
$$

The precise nature of the Optimality conditions depends on the utility function. If $U\left(x_{1}\right)>$ $U\left(x_{2}\right)$, the condition is,

$$
s_{11}^{1} \geq \frac{1}{2} \text { and } s_{22}^{2} \leq \frac{1}{2},
$$

with at least one strict. Substitution for $s_{11}^{1}$ yields,

$$
p_{1}^{g} \geq \frac{\left(1-\mu^{g}\right) p_{1}^{h}}{\mu^{g}}=\frac{p_{1}^{h}}{R}
$$

where,

$$
R \equiv \frac{\mu^{g}}{1-\mu^{g}}
$$

Substitution for $s_{22}^{2}$ yields,

$$
1-p_{1}^{g} \leq \frac{\left(1-\mu^{g}\right)\left(1-p_{1}^{h}\right)}{\mu^{g}}=\frac{1-p_{1}^{h}}{R},
$$

or,

$$
p_{1}^{g} \geq \frac{R-1}{R}+\frac{p_{1}^{h}}{R}
$$


Looking at these necessary conditions one can identify three cases depending on the value of $R$ : if $R=1\left(\mu^{g}=\frac{1}{2}\right)$, then in order for both inequalities to hold, one strictly, it is necessary and sufficient that $p_{1}^{g}>p_{1}^{h}$; if $R>1\left(\mu^{g}>\frac{1}{2}\right)$, then the only constraint is $p_{1}^{g} \geq \frac{R-1}{R}+\frac{p_{1}^{h}}{R}$; and finally, if $R<1\left(\mu^{g}<\frac{1}{2}\right)$, the only constraint is $p_{1}^{g} \geq \frac{p_{1}^{h}}{R}$. The conditions in the case in which $U\left(x_{2}\right)>U\left(x_{1}\right)$ are precisely the converse. Hence combining the two permissible utility functions, we arrive at the following necessary and sufficient conditions:

- If $R=1\left(\mu^{g}=\frac{1}{2}\right)$, then,

$$
p_{1}^{g} \neq p_{1}^{h}
$$

- If $R>1\left(\mu^{g}>\frac{1}{2}\right)$, then the constraints are,

$$
p_{1}^{g} \geq \frac{R-1}{R}+\frac{p_{1}^{h}}{R} \text { or } p_{1}^{g} \leq \frac{p_{1}^{h}}{R} .
$$

- If $R<1\left(\mu^{g}<\frac{1}{2}\right)$, then the constraints are,

$$
p_{1}^{g} \geq \frac{p_{1}^{h}}{R} \text { or } p_{1}^{g} \leq \frac{R-1}{R}+\frac{p_{1}^{h}}{R} .
$$

\subsection{Two Examples}

Example 1 Consider the case with $\mu^{g}=0.8$ so that $R=4$. If $U\left(x_{1}\right)>U\left(x_{2}\right)$, the condition is,

$$
p_{1}^{g} \geq 0.75+0.25 p_{1}^{h}
$$

Conversely, if $U\left(x_{2}\right)>U\left(x_{1}\right)$, the condition is,

$$
p_{1}^{g} \leq 0.25 p_{1}^{h}
$$

We draw these constraint sets in figure 3.

Example 2 The model can characterize an apparent reversal of preference, as when $\mu^{g}=0.5$ and stochastic action choice is,

$$
p_{1}^{g}=0.9 ; p_{1}^{h}=0.7
$$

so that $x_{1}$ is chosen with probability 0.9 with layout $g$, yet only with probability 0.3 with layout $h$. To construct a RE-PREP in this case, set $U\left(x_{1}\right)>U\left(x_{2}\right)$ and allow as usual for one mental state $s^{1}$ in which $y_{1}$ is taken and another mental state $s^{2}$ in which $y_{2}$ is taken so that, by Data Matching,

$$
\rho^{g}\left(s^{1}\right)=0.9 \text { and } \rho^{h}\left(s^{1}\right)=0.7
$$




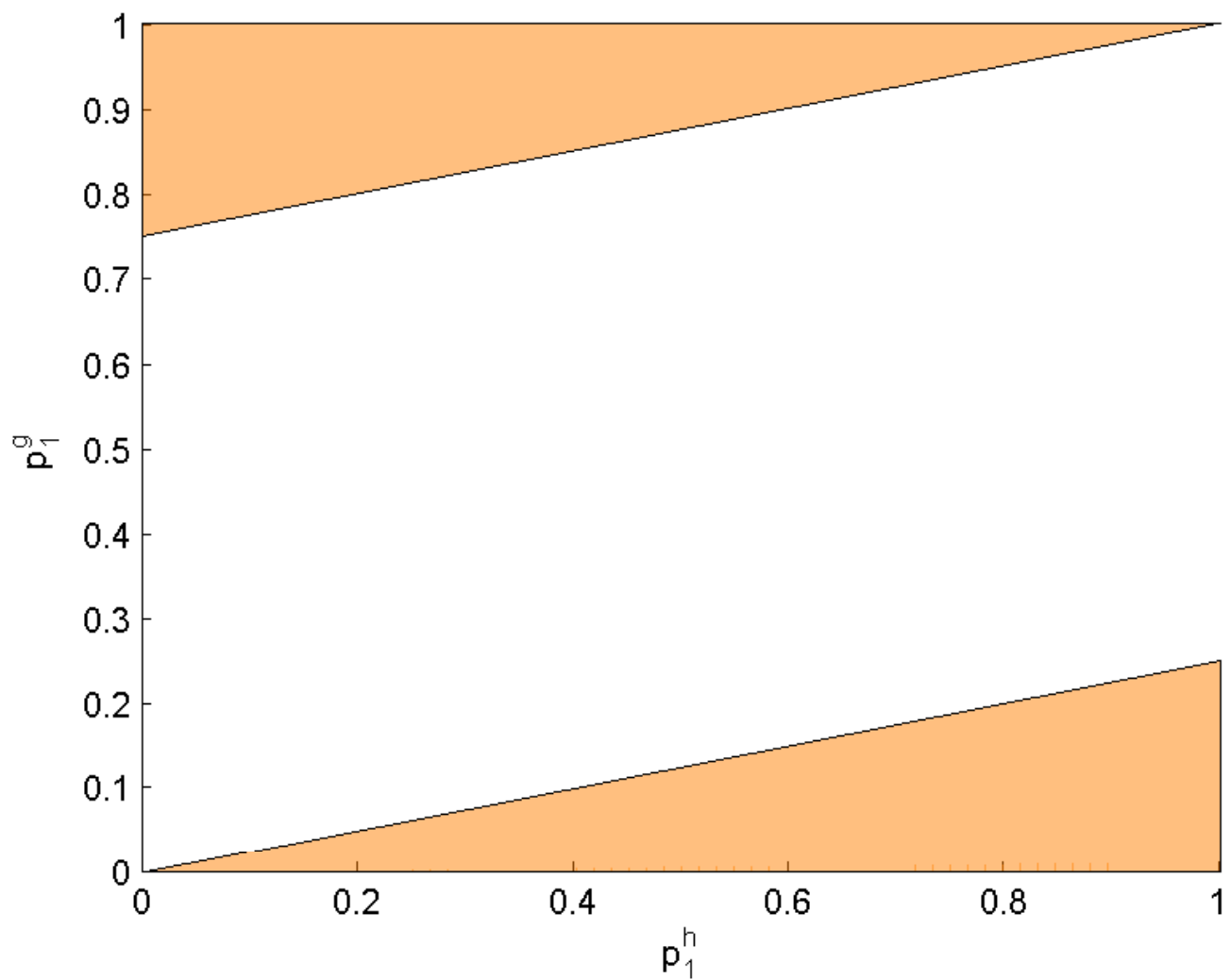

Figure 3: Constraints implied by $\mathrm{R}=4$ (interior region is infeasible) 
This implies that the lottery associated with choice of $y_{1}$ in $s^{1}$ is yields prize $x_{1}$ with probability,

$$
\frac{0.9}{0.9+0.7}>0.5
$$

Similarly, the prize lottery associated with action $y_{2}$ in $s^{2}$ yields $x_{1}$ with probability,

$$
\frac{0.3}{0.1+0.3}>0.5 \text {. }
$$

We complete the specification of a RE-PREP by applying the Rational Expectations condition to set the probabilities for the lotteries associated with the untaken actions in line with what they would actually be,

$$
\begin{aligned}
s_{21}^{1} & =\frac{0.5 \rho^{h}\left(s^{1}\right)}{0.5 \rho^{g}\left(s^{1}\right)+0.5 \rho^{h}\left(s^{1}\right)}=\frac{0.7}{0.9+0.7}<0.5 \\
s_{11}^{2} & =\frac{0.5\left(1-\rho^{g}\left(s^{1}\right)\right)}{0.5\left(1-\rho^{g}\left(s^{1}\right)\right)+0.5\left(1-\rho^{h}\left(s^{1}\right)\right)}=\frac{0.1}{0.1+0.3}<0.5 .
\end{aligned}
$$

Hence it is strictly optimal to pick $y_{1}$ in $s^{1}$ and $y_{2}$ in $s^{2}$, completing the confirmation that this is a $R E-P R E P$.

\section{No Improving Action Switches}

\subsection{Blind Switches and Action Switches}

A simple idea organizes the conditions identified in the $2 \times 2 \times 2$ case and applies to the general case. First, one must look through the actions that are chosen to the stochastic demand for prizes that results. Consider example 1 above in this light, with $\mu^{g}=0.8$ so that $R=4$, and suppose that $p_{1}^{g}=0.75$ and $p_{1}^{h}=0$ so that $1-p_{1}^{g}=0.25$ and $1-p_{1}^{h}=1$. In this case, whenever $y_{1}$ is taken, the layout is $g$, so that it yields prize $x_{1}$ for sure. On the other hand, act $y_{2}$ is taken both when it yields prize $x_{1}$ (layout $h$ ) and when it yields prize $x_{2}$ (layout $g$ ). To understand the stochastic demand for prizes resulting from action $y_{2}$, note that, conditional on this action being taken, the layout is equally likely to be $g$ or $h$,

$$
\frac{\mu^{g}\left(1-p_{1}^{g}\right)}{\mu^{g}\left(1-p_{1}^{g}\right)+\left(1-\mu^{g}\right)\left(1-p_{1}^{h}\right)}=\frac{\left.1-\mu^{g}\right)\left(1-p_{1}^{h}\right)}{\left(1-\mu^{g}\right)\left(1-p_{1}^{h}\right)}=0.5
$$

resulting in an overall even chance of winning prize $x_{1}$ or prize $x_{2}$.

One general point to note about this example is that if $U\left(x_{1}\right)>U\left(x_{2}\right)$, the overall probability of getting the good prize is 1 in the $60 \%$ of cases in which action $y_{1}$ is taken, and 0.5 in the $40 \%$ of 
cases in which action $y_{2}$ is taken, for a net probability of 0.8 . It is intuitive that there cannot be a RE-PREP with a lower probability of getting the best prize, since the simple strategy of always picking $y_{1}$ yields the same outcome. This illustrates the "No Improving Blind Switches" (NIBS) condition that we show in the section 5 to be necessary for a RE-PREP.

There is a second observation that explains the constraints with more precision. Note that the chance of getting the better prize from action $y_{2}$ cannot be strictly less than $50 \%$. Intuitively, if it were to be thus, then it would be strictly superior to switch action $y_{2}$ to $y_{1}$ in state $s^{1}$, so as to always take the action $y_{1}$. This is the No Improving Action Switches (NIAS) condition that we show in the next section to fully characterize a RE-PREP.

To apply the NIAS condition to the current case, note that the probability of receiving the good prize when action $y_{1}$ is taken is the conditional probability that the layout is $g$ when $y_{1}$ is taken,

$$
\frac{\mu^{g} p_{1}^{g}}{\mu^{g} p_{1}^{g}+\left(1-\mu^{g}\right) p_{1}^{h}}
$$

The probability of receiving the good prize if action $y_{2}$ had been taken every time that action $y_{1}$ was taken would be the converse,

$$
\frac{\left(1-\mu^{g}\right) p_{1}^{h}}{\mu^{g} p_{1}^{g}+\left(1-\mu^{g}\right) p_{1}^{h}}
$$

Hence the condition that this not yield an improvement is,

$$
\mu^{g} p_{1}^{g} \geq\left(1-\mu^{g}\right) p_{1}^{h}
$$

or,

$$
p_{1}^{g} \geq \frac{p_{1}^{h}}{R}
$$

Similarly, the condition under which action $y_{2}$ should not be globally replaced by action $y_{1}$ is,

$$
\left(1-\mu^{g}\right)\left(1-p_{1}^{h}\right) \geq \mu^{g}\left(1-p_{1}^{g}\right)
$$

or,

$$
p_{1}^{g} \geq \frac{R-1}{R}+\frac{p_{1}^{h}}{R}
$$

In the current case with $\mu^{g}=0.8$ and $R=4$, the only binding inequality is,

$$
p_{1}^{g} \geq 0.75+0.25 p_{1}^{h}
$$

The fact that this is identical to the constraint identified in example 1 for the case $U\left(x_{1}\right)>U\left(x_{2}\right)$ (the upper left shaded region in figure 3) is not coincidental. 


\subsection{The NIAS Inequalities}

The above example pinpoints the central concept in our representation theorem, which involves ruling out the existence of improving action switches.

Definition 2 Utility function $U: X \rightarrow \mathbb{R}$ satisfies the $\boldsymbol{N I A S}$ inequalities with respect to $(X, Y, \mu, P)$ if for all $k, m \in\{1, . ., M\}$,

$$
\sum_{f \in \mathcal{F}} \mu(f) P^{f}\left(y_{m}\right)\left[U\left(f\left(y_{m}\right)\right)-U\left(f\left(y_{k}\right)\right] \geq 0,\right.
$$

with at least one inequality strict.

Note that the NIAS inequalities can be expressed in an alternative form that is more readily compatible with the RE condition. In particular, given $(X, Y, \mu, P)$ and $y_{m} \in Y$ such that $P^{f}\left(y_{m}\right)>$ 0 for some $f \in \mathcal{F}(\mu)$, the stochastic demand for prizes associated with choosing $y_{k}$ in place of $y_{m}$, $\delta_{m k} \in \Delta(X)$ can be defined as,

$$
\delta_{m k}\left(x_{n}\right)=\frac{\sum_{\left\{f \in \mathcal{F} \mid f\left(y_{k}\right)=x_{n}\right\}} \mu(f) P^{f}\left(y_{m}\right)}{\sum_{f \in \mathcal{F}} \mu(f) P^{f}\left(y_{m}\right)} .
$$

It is direct that the utility function $U: X \rightarrow \mathbb{R}$ satisfies the NIAS inequalities with respect to $(X, Y, \mu, P)$ if, for all $y_{m} \in Y$ such that $P^{f}\left(y_{m}\right)>0$ for some $f \in \mathcal{F}(\mu)$, and for all $k \in\{1, \ldots, M\}$,

$$
\sum_{n=1}^{N}\left[\delta_{m m}\left(x_{n}\right)-\delta_{m k}\left(x_{n}\right)\right] U\left(x_{n}\right) \geq 0,
$$

with at least one inequality strict.

The key observation is that a necessary and sufficient condition for a RE-PREP is that there exists a utility function such that the NIAS inequalities hold, so that for every taken action, it is better not to switch to taking some fixed alternative action in all situations in which the given action was taken. Note that $\delta_{m m} \in \Delta(X)$ is the stochastic demand associated with choice of $y_{m}$ in the IDS. Note also that the NIAS inequalities correspond to non-emptiness of the intersection of $(M-1)^{2}$ linear inequalities, which can be identified using standard linear programming methods.

\subsection{Characterization}

Theorem $1(X, Y, \mu, P)$ have a $R E$-PREP representation if and only if there exists $U: X \rightarrow \mathbb{R}$ satisfying the NIAS inequalities. 
Proof. Sufficiency: We identify a RE-PREP with exactly one state of mind $s^{j}$ for each action $y_{j}$ that is taken with strictly positive probability, so that,

$$
C\left(s^{j}\right)=y_{j}
$$

We set $\rho^{f}\left(s^{j}\right)=P^{f}\left(y_{j}\right)$ for all $f \in \mathcal{F}(\mu)$ and $j \in\{1, \ldots, M\}$, and pick $U: X \rightarrow \mathbb{R}$ satisfying the NIAS inequalities. We note that, by construction, $(\rho, C)$ satisfy Data Matching. Moreover, given $y_{j} \in Y$ with $P^{f}\left(y_{j}\right)>0$, we use the Rational Expectations condition to solve for all $s_{m n}^{j}$ : for all $m \in\{1, \ldots, M\}, n \in\{1, \ldots, N\}$,

$$
s_{m n}^{j}=\frac{\sum_{\left\{f \in \mathcal{F} \mid f\left(y_{m}\right)=x_{n}\right\}} \mu(f) P^{f}\left(y_{j}\right)}{\sum_{f \in \mathcal{F}} \mu(f) P^{f}\left(y_{j}\right)} .
$$

Finally, note that, upon substitution for $s_{m n}^{m}$ and $s_{k n}^{m}$ as above, we derive the conditions for Optimality as, for all $k \in\{1, \ldots, M\}$,

$$
\sum_{n=1}^{N}\left[\frac{\sum_{\left\{f \in \mathcal{F} \mid f\left(y_{m}\right)=x_{n}\right\}} \mu(f) P^{f}\left(y_{m}\right)}{\sum_{f \in \mathcal{F}} \mu(f) P^{f}\left(y_{m}\right)}\right] U\left(x_{n}\right) \geq \sum_{n=1}^{N}\left[\frac{\sum_{\left\{f \in \mathcal{F} \mid f\left(y_{k}\right)=x_{n}\right\}} \mu(f) P^{f}\left(y_{m}\right)}{\sum_{f \in \mathcal{F}} \mu(f) P^{f}\left(y_{m}\right)}\right] U\left(x_{n}\right),
$$

with at least one strict. This reduces precisely to the NIAS inequalities that $U$ satisfies by construction.

Necessity: A direct review of the above logic shows that identifying a utility function that satisfies the NIAS inequalities is not only sufficient for a RE-PREP, but also necessary for a RE-PREP in which there is only one state of mind per action. The full result follows from the observation that if we identify a RE-PREP with more than one mental state for one or more action choices, then there must exist a RE-PREP with only one mental state for each action choice: allowing for multiple states does not expand the set of IDS for which a RE-PREP exists.

Suppose that we have identified a RE-PREP $(\rho, C, U)$ of $(X, Y, \mu, P)$ such that, for all actions $y_{j}$ taken with strictly positive probability, there exists states $s^{j, p} \in S(\rho)$ for $p \in\{1, \ldots, P(j)\}$ with,

$$
C\left(s^{j, p}\right)=y_{j} \text { for all } p
$$

and with $P(j)>1$ for some $j$.

Define a new triple $(\tilde{\rho}, \tilde{C}, U)$ that is identical to $(\rho, C, U)$ except in the two respects. First, for all $j \in\{1, \ldots, M\}$ such that action $y_{j}$ is taken with strictly positive probability, set $\tilde{C}\left(y_{j}\right)=\tilde{s}^{j}$, where the prize lottery corresponding to state $\tilde{s}^{j}$ is defined as,

$$
\tilde{s}_{m n}^{j}=\frac{\sum_{p=1}^{P(j)} \sum_{\left\{f \in \mathcal{F} \mid f\left(y_{m}\right)=x_{n}\right\}} \mu(f) \rho^{f}\left(s^{j, p}\right)}{\sum_{p=1}^{P(j)} \sum_{\{f \in \mathcal{F}\}} \mu(f) \rho^{f}\left(s^{j, p}\right)} .
$$


Second, define $\tilde{\rho}$ so that $\tilde{s}^{j}$ is perceived in place of all $s^{j, p}$,

$$
\tilde{\rho}^{f}\left(\tilde{s}^{j}\right)=\sum_{p=1}^{P(j)} \rho^{f}\left(s^{j, p}\right)
$$

By construction, note that $\tilde{C}^{-1}\left(y_{j}\right)$ is a singleton with a single state $\tilde{s}^{j}$ replacing all $s^{j, p} \in S(\rho)$,

$$
\tilde{C}^{-1}\left(y_{j}\right)=\tilde{s}^{j}
$$

To show that $(\tilde{\rho}, \tilde{C}, U)$ form a RE-PREP of $(X, Y, \mu, P)$, note first that $(\tilde{\rho}, \tilde{C}, U)$ satisfies Data Matching and Rational Expectations by construction. Optimality is established by noting that it survives under convex combinations. What must be shown is that,

$$
\sum_{n=1}^{N} \tilde{s}_{m n}^{m} U\left(x_{n}\right) \geq \sum_{n=1}^{N} \tilde{s}_{m n}^{k} U\left(x_{n}\right) \text { for all } m, k \in\{1, \ldots, M\} .
$$

Upon substitution for $\tilde{s}_{m n}^{m}$ and $\tilde{s}_{k n}^{m}$ and after removing the common denominator, the Optimality conditions become, for all $m, k \in\{1, \ldots, M\}$,

$$
\sum_{n=1}^{N}\left[\sum_{p=1}^{P(m)} \sum_{\left\{f \in \mathcal{F} \mid f\left(y_{m}\right)=x_{n}\right\}} \mu(f) \rho^{f}\left(s^{m, p}\right)\right] U\left(x_{n}\right) \geq \sum_{n=1}^{N}\left[\sum_{p=1}^{P(m)} \sum_{\left\{f \in \mathcal{F} \mid f\left(y_{k}\right)=x_{n}\right\}} \mu(f) \rho^{f}\left(s^{m, p}\right)\right] U\left(x_{n}\right),
$$

or,

$$
\sum_{p=1}^{P(m)} \sum_{n=1}^{N} \sum_{\left\{f \in \mathcal{F} \mid f\left(y_{m}\right)=x_{n}\right\}} \mu(f) \rho^{f}\left(s^{m, p}\right) U\left(x_{n}\right) \geq \sum_{p=1}^{P(m)} \sum_{n=1}^{N} \sum_{\left\{f \in \mathcal{F} \mid f\left(y_{k}\right)=x_{n}\right\}} \mu(f) \rho^{f}\left(s^{m, p}\right) U\left(x_{n}\right) .
$$

Validity of these conditions follows immediately from the fact that $(\rho, C, U)$ form a RE-PREP of $(X, Y, \mu, P)$, so that the Optimality condition implies that $y_{m}$ is a utility maximizer for each state $s^{m, p} \in S(\rho)$,

$$
\sum_{n=1}^{N} \sum_{\left\{f \in \mathcal{F} \mid f\left(y_{m}\right)=x_{n}\right\}} \mu(f) \rho^{f}\left(s^{m, p}\right) U\left(x_{n}\right) \geq \sum_{n=1}^{N} \sum_{\left\{f \in \mathcal{F} \mid f\left(y_{k}\right)=x_{n}\right\}} \mu(f) \rho^{f}\left(s^{m, p}\right) U\left(x_{n}\right) .
$$

Note from the above proof that a necessary condition for $(\rho, C, U)$ to comprise a RE-PREP of $(X, Y, \mu, P)$ is that $U$ satisfy the NIAS conditions. 


\section{$5 \quad$ Two Framing Effects}

\subsection{Layout Effects}

The characterization theorem allows for cases in which the layout has significant impact on stochastic demand - all that is necessary is that the NIAS conditions are satisfied. Hence there may be two frames $f, g \in F$ and $x \in X$ such that,

$$
P^{f}(\{y \in Y \mid f(y)=x\}) \neq P^{g}(\{y \in Y \mid g(y)=x\}) .
$$

Consider for example top-down search in a list (see Rubinstein and Salant [2006]). In terms of perception, this may plausibly result in greater clarity about objects high in the list than those further down the list, which may in turn make them more likely to be chosen (as in Geng [2011]), and also more likely to be of high utility when so chosen. The following stark example illustrates these effects at work.

Example 3 Suppose that there are 4 prizes, one of each value $\$ 1$ through $\$ 4$, and that each of the four choices is ex ante equally likely to yield each prize:

$$
\begin{aligned}
X & =\{\$ 1, \$ 2, \$ 3, \$ 4\} ; \\
Y & =\left\{y_{m} \mid 1 \leq m \leq 4\right\} ; \\
\mu(f) & =\frac{1}{4 !} \text { all } f \in \mathcal{F} .
\end{aligned}
$$

Now suppose that the process of perception is such that the prize corresponding to action 1 (the top line of four) is always seen with perfect clarity, while the other actions are not understood beyond the prior and the identify of the prize corresponding to $y_{1}$. In this case, there are 4 subjective states,

$$
S(\rho)=\left\{\left(x, \Lambda_{-x}\right) \in X \times \Delta(X)^{3}\right\}
$$

where $\Lambda_{-x}$ assigns to each $y_{m}$ with $m>1$ the lottery that offers all prizes in $X / x$ with probability $\frac{1}{3}$. If the DM is risk neutral, action $y_{1}$ will be chosen whenever it is seen to contain a prize of $\$ 3$ or \$4, which is a 50\% chance, with the other options dividing up the remaining probability. In this case, note that selection of $y_{1}$ can give rise only to prizes $\$ 3$ and $\$ 4$, with equal probability, while for $y_{n}$ with $n>1$, each action can give rise to any prize, with a one-third chance of either the \$3 or the $\$ 4$ prize, and a one-sixth chance of either the \$1 or the \$2 prize. 


\subsection{Informational Effects}

When the impact of imperfect perception on stochastic choice is considered by economists, it is typically treated as resulting from similarities between product characteristics (e.g. Debreu [1960] and Natenzon [2011]). In such cases the precise layout has no impact on stochastic choice of prizes, so standard framing effects are not present. Yet even in such cases, the NIAS inequalities imply that the layout-generating mechanism $\mu$ may impact prize choice, since any RE-PREP of $(X, Y, \mu, P)$ must respect the information that the $\mu$ provides on prize location.

The No Improving Blind Switches (NIBS) inequalities mentioned in section 3 provide insight into how the layout-generating mechanism impacts choice of prize. These inequalities summarize the utility available to an agent who always makes the same choice of action. We show in theorem 2 that these simple inequalities on $U: X \rightarrow \mathbb{R}$ are necessary for $(\rho, C, U)$ to provide a RE-PREP representation of $(X, Y, \mu, P)$.

Definition 3 Utility function $U: X \rightarrow \mathbb{R}$ satisfies the $\mathbf{N I B S}$ inequalities with respect to $(X, Y, \mu, P)$ if the chosen actions yield at least as high utility as would always choosing any fixed action $y_{k} \in Y$,

$$
\sum_{f \in \mathcal{F}} \sum_{y_{m} \in Y} \mu(f) P^{f}\left(y_{m}\right) U\left(f\left(y_{m}\right)\right) \geq \max _{y_{k} \in Y} \sum_{f \in \mathcal{F}} \mu(f) U\left(f\left(y_{k}\right)\right)
$$

with strict inequality for some $y_{k} \in Y$.

Theorem 2 If $(\rho, C, U)$ provide a $R E$-PREP representation of $(X, Y, \mu, P)$, then $U: X \rightarrow \mathbb{R}$ satisfies the NIBS inequalities.

Proof. As noted directly after the proof of theorem 1 , if $(\rho, C, U)$ provide a RE-PREP representation of $(X, Y, \mu, P)$, then $U: X \rightarrow \mathbb{R}$ must satisfy the NIAS inequalities. We show now that NIAS implies NIBS. There are two cases:

1. Suppose first that $U: X \rightarrow \mathbb{R}$ satisfies the NIAS inequalities, yet that there exists $y_{k} \in Y$ such that,

$$
\sum_{f \in \mathcal{F}} \mu(f) U\left(f\left(y_{k}\right)\right)>\sum_{f \in \mathcal{F}} \sum_{y_{m} \in Y} \mu(f) P^{f}\left(y_{m}\right) U\left(f\left(y_{m}\right)\right) .
$$

Substitute $\sum_{y_{m} \in Y} P^{f}\left(y_{m}\right)=1$ on the LHS and rearrange to conclude that,

$$
\sum_{y_{m} \in Y} \sum_{f \in \mathcal{F}} \mu(f) P^{f}\left(y_{m}\right) U\left(f\left(y_{k}\right)\right)>\sum_{y_{m} \in Y} \sum_{f \in \mathcal{F}} \mu(f) P^{f}\left(y_{m}\right) U\left(f\left(y_{m}\right)\right),
$$


which in turn implies the existence of at least one possible action $y_{m} \neq y_{k} \in Y$ such that replacing $y_{m}$ by $y_{k}$ strictly increases utility,

$$
\sum_{f \in \mathcal{F}} \mu(f) P^{f}\left(y_{m}\right) U\left(f\left(y_{k}\right)\right)>\sum_{f \in \mathcal{F}} \mu(f) P^{f}\left(y_{m}\right) U\left(f\left(y_{m}\right)\right)
$$

directly in contradiction to the NIAS inequalities.

2. Now suppose that $U: X \rightarrow \mathbb{R}$ satisfies the NIAS inequalities, yet for all $y_{k} \in Y$,

$$
\sum_{f \in \mathcal{F}} \mu(f) U\left(f\left(y_{k}\right)\right)=\sum_{f \in \mathcal{F}} \sum_{y_{m} \in Y} \mu(f) P^{f}\left(y_{m}\right) U\left(f\left(y_{m}\right)\right) .
$$

Substitute $\sum_{y_{m} \in Y} P^{f}\left(y_{m}\right)=1$ on the LHS and rearrange to conclude that,

$$
\sum_{y_{m} \in Y} \sum_{f \in \mathcal{F}} \mu(f) P^{f}\left(y_{m}\right) U\left(f\left(y_{k}\right)\right)=\sum_{y_{m} \in Y} \sum_{f \in \mathcal{F}} \mu(f) P^{f}\left(y_{m}\right) U\left(f\left(y_{m}\right)\right) .
$$

Noting that the above holds for all $y_{k} \in Y$, we conclude that either there exists $y_{k}, y_{m} \in Y$ such that,

$$
\sum_{f \in \mathcal{F}} \mu(f) P^{f}\left(y_{m}\right) U\left(f\left(y_{k}\right)\right)>\sum_{f \in \mathcal{F}} \mu(f) P^{f}\left(y_{m}\right) U\left(f\left(y_{m}\right)\right)
$$

or for all $y_{k}, y_{m} \in Y$,

$$
\sum_{f \in \mathcal{F}} \mu(f) P^{f}\left(y_{m}\right) U\left(f\left(y_{k}\right)\right)=\sum_{f \in \mathcal{F}} \mu(f) P^{f}\left(y_{m}\right) U\left(f\left(y_{m}\right)\right) .
$$

The former contradicts the weak inequality in NIAS, while the latter contradicts the need for strict inequality in at least one case.

The necessity of the NIBS inequalities is intuitive, given that the Rational Expectations assumption requires the DM to fully understand the statistical association between subjective perceptions, choices, and prizes in a RE-PREP. These inequalities provide a particularly simple measure of the information content of $\mu$. They directly tie the degree of asymmetry in the layout-generating mechanism to the class of utility functions for which a RE-PREP exists. With a completely asymmetric layout-generating mechanism in which a given action always produces the same prize, the NIBS inequalities imply that all actions that are ever chosen must yield maximal utility. These inequalities are less exacting when the layout-generating mechanism is completely symmetric, so that blind choice of any action results in all prizes being equiprobable. In such a case, the NIBS inequalities 
imply only that overall stochastic demand for prizes yields above the simple unweighted average level of utility.

The NIBS inequalities have important behavioral implications. They point out that the need for perceptual attention depends on the layout-generating mechanism. In the extreme case, a participant in a multiple-choice test could score $100 \%$ without the slightest need to read the questions if the first answer was always correct. What this means is that attentional effort is likely to be a function of the layout-generating mechanism. The experiment in section 7 presents preliminary evidence that this is indeed so - even in less extreme cases.

\section{Imperfect Perception and Stochastic Choice}

\subsection{Stochastic Choice Data}

It is rare in applied settings to have easy access to information on either action choices or the layout-generating mechanism. For this reason, the standard data set for stochastic choice is the pattern of observed demand for prizes across all choice problems. In this section, we characterize restrictions on just such standard stochastic choice data associated with the existence of RE-PREP representations based on a fixed utility function.

Definition 4 A stochastic choice system $(X, Q)$ comprises a finite grand set $X$ of prizes and a correspondence,

$$
Q: \mathcal{X} \longrightarrow \Delta(\mathcal{X})
$$

with for all $A \in \mathcal{X}$,

$$
Q^{A}(x)>0 \Longrightarrow x \in A
$$

where $\mathcal{X}$ is the set of all non-empty subsets of $X$.

\subsection{RE-PREPs}

We look for a representation of the entire choice system that involves a fixed utility function but a variety of action choice subsets. We begin by specifying some action choice set $Y$, noting that it may be conceptual rather than actually observed. For simplicity, we assume that $Y$ has the same 
cardinality as $X$. We specify also choice sets $Y_{A} \subset Y$ with $\left|Y_{A}\right|=|A|$, corresponding to each set $A \in \mathcal{X}$, as well as $\mathcal{F}_{A}$, the class of all 1-1 and onto functions from $Y_{A}$ to $A$. The existence of a REPREP then hinges on whether or not one can construct ideal data sets $P_{A}: \mathcal{F}_{A}\left(\mu_{A}\right) \longrightarrow \Delta\left(Y_{A}\right)$; measures $\mu_{A} \in \Delta\left(\mathcal{F}_{A}\right)$; perception functions $\rho_{A}: \mathcal{F}_{A} \longrightarrow S_{A}$; and choice functions $C_{A}: S_{A} \longrightarrow Y_{A}$ with $S_{A}=\Delta(X)^{|A|}$; such that there exist RE-PREPs $\left(A, Y_{A}, \mu_{A}, P_{A}\right)$ of the form $\left(\rho_{A}, C_{A}, U\right)$ in which the stochastic choice system is reproduced. Note that the sets $Y_{A}$ and $Y$ are essentially arbitrary.

Definition 5 Stochastic choice system $(X, Q)$ has a $\boldsymbol{R} \boldsymbol{E}-\boldsymbol{P R} \boldsymbol{E P}$ representation if there exists $Y$ with $|Y|=|X|, U: X \longrightarrow \mathbb{R}$, and quadruples $\left(A, Y_{A}, \mu_{A}, P_{A}\right)$ for all $A \in \mathcal{X}$, with $Y_{A} \subset Y$; $\left|Y_{A}\right|=|A| ; \mu_{A} \in \Delta\left(\mathcal{F}_{A}\right) ;$ and $P_{A}: \mathcal{F}_{A} \longrightarrow \Delta\left(Y_{A}\right)$; all of which have RE-PREP representations $\left(\rho_{A}, C_{A}, U\right)$ with,

$$
Q^{A}\left(x_{n}\right)=\sum_{f \in \mathcal{F}_{A}} \mu_{A}(f) P_{A}^{f}\left(f^{-1}\left(x_{n}\right)\right) \text { for all } x_{n} \in A,
$$

where the inverse function $f^{-1}: A \rightarrow Y_{A}$ is defined by $f\left(f^{-1}\left(x_{n}\right)\right)=x_{n}$.

\subsection{The Representation Theorem}

The general characterization theorem captures the intuition that a RE-PREP representation exists provided one can find a utility function such that expected utility in any choice problem $A \in \mathcal{X}$ is strictly higher than that associated with blind choice in $A$.

Theorem 3 Stochastic choice system $(X, Q)$ has a RE-PREP representation if and only if there exists $U: X \rightarrow \mathbb{R}$ such that, for all $A \in \mathcal{X}$,

$$
\sum_{x_{n} \in A} Q^{A}\left(x_{n}\right) U\left(x_{n}\right)>\sum_{x_{n} \in A} \frac{U\left(x_{n}\right)}{|A|} .
$$

Proof. Sufficiency: Define the IDS so that it always gives rise to the given stochastic demand,

$$
P_{A}^{f}(y)=Q^{A}(f(y))
$$

for all $f \in \mathcal{F}_{A}$ and $y \in Y_{A}$, and define $\mu_{A}$ is a symmetric measure assigning equal probability $\frac{1}{|A| !}$ to each $f \in \mathcal{F}_{A}$. With these two strong symmetry conditions, a direct counting argument shows that 
the stochastic demand for prizes associated with replacing $y_{m}$ with $y_{k} \neq y_{m}$ is independent of $y_{m}$ and $y_{k}$. Specifically, the probability of getting prize $x_{n} \in A$ with any such switch is,

$$
\delta_{m k}^{A}\left(x_{n}\right)=\frac{1-Q^{A}\left(x_{n}\right)}{|A|-1} .
$$

With this strong symmetry among chosen and unchosen acts, there is one and only one inequality for the NIAS condition to be satisfied:

$$
\sum_{x_{n} \in A} Q^{A}\left(x_{n}\right) U\left(x_{n}\right)>\sum_{x_{n} \in A}\left[\frac{1-Q^{A}\left(x_{n}\right)}{|A|-1}\right] U\left(x_{n}\right),
$$

or,

$$
\sum_{x_{n} \in A} Q^{A}\left(x_{n}\right) U\left(x_{n}\right)>\sum_{x_{n} \in A} \frac{U\left(x_{n}\right)}{|A|},
$$

as assumed. Hence for all $A \in \mathcal{X}$, the NIAS conditions hold for $\left(A, Y_{A}, \sigma_{A}, P_{A}\right)$, which therefore possesses a RE-PREP representation with the fixed utility function $U: X \rightarrow \mathbb{R}$. Hence $(X, Q)$ has a symmetric RE-PREP representation.

Necessity: Suppose that the condition is not satisfied, but that $(X, Q)$ has a RE-PREP representation for some fixed utility function $U: X \rightarrow \mathbb{R}$. We establish a contradiction by showing that $U$ does not satisfy the NIBS inequalities. By assumption, there exists $A \in \mathcal{X}$ such that,

$$
\sum_{x_{n} \in A} Q^{A}\left(x_{n}\right) U\left(x_{n}\right) \leq \sum_{x_{n} \in A} \frac{U\left(x_{n}\right)}{|A|} .
$$

Substitution for $Q^{A}\left(x_{n}\right)$ yields,

$$
\sum_{x_{n} \in A} \sum_{f \in \mathcal{F}_{A}} \mu_{A}(f) P_{A}^{f}\left(f^{-1}\left(x_{n}\right)\right) U\left(x_{n}\right) \leq \sum_{x_{n} \in A} \frac{U\left(x_{n}\right)}{|A|} .
$$

Note that the LHS of $(*)$ is simply the expected utility of the chosen acts,

$$
\sum_{x_{n} \in A} \sum_{f \in \mathcal{F}_{A}} \mu_{A}(f) P_{A}^{f}\left(f^{-1}\left(x_{n}\right)\right) U\left(x_{n}\right)=\sum_{f \in \mathcal{F}_{A}} \sum_{y_{m} \in Y_{A}} \mu_{A}(f) P_{A}^{f}\left(y_{m}\right) U\left(f\left(y_{m}\right)\right) .
$$

We now show the relationship between the RHS of $(*)$ and blind choices. Summation across blind choices yields,

$$
\sum_{y_{k} \in Y_{A}} \sum_{f \in \mathcal{F}_{A}} \mu_{A}(f) U\left(f\left(y_{k}\right)\right)=\sum_{x_{n} \in A} \sum_{f \in \mathcal{F}_{A}} \mu_{A}(f) U\left(x_{n}\right)=\sum_{x_{n} \in A} U\left(x_{n}\right) .
$$

There are two cases to consider: 
1. If all blind choices yield the same amount, (**) implies that they all yield simple arithmetic average utility,

$$
\sum_{f \in \mathcal{F}_{A}} \mu_{A}(f) U\left(f\left(y_{k}\right)\right)=\sum_{x_{n} \in A} \frac{U\left(x_{n}\right)}{|A|} \text { for all } y_{k} \in Y_{A} .
$$

Substitution as indicated for LHS and RHS in (*) then yields,

$$
\sum_{f \in \mathcal{F}_{A}} \sum_{y_{m} \in Y_{A}} \mu_{A}(f) P_{A}^{f}\left(y_{m}\right) U\left(f\left(y_{m}\right)\right) \leq \sum_{f \in \mathcal{F}_{A}} \mu_{A}(f) U\left(f\left(y_{k}\right)\right)
$$

for all $y_{k} \in Y$. This directly contradicts NIBS, which requires strict inequality in the other direction.

2. If not all blind choices yield the same amount of utility, (**) implies that there exists $y_{k} \in Y_{A}$ yielding strictly above simple arithmetic average utility from blind choice,

$$
\sum_{f \in \mathcal{F}_{A}} \mu_{A}(f) U\left(f\left(y_{k}\right)\right)>\sum_{x_{n} \in A} \frac{U\left(x_{n}\right)}{|A|} .
$$

Substitution as indicated for LHS and RHS in $(*)$ then yields,

$$
\sum_{f \in \mathcal{F}_{A}} \sum_{y_{m} \in Y_{A}} \mu_{A}(f) P_{A}^{f}\left(y_{m}\right) U\left(f\left(y_{m}\right)\right)<\sum_{f \in \mathcal{F}_{A}} \mu_{A}(f) U\left(f\left(y_{k}\right)\right),
$$

which directly contradicts NIBS.

\subsection{Imperfect Perception or Stochastic Utility?}

For more than fifty years, the dominant theory of stochastic choice has involved placing all randomness in the utility function (see Luce [1959] and Block and Marschak [1960]). The most famous axiom for such cases is Luce's axiom, whereby the ratio of the probabilities of choosing any one item over any other is independent of other "irrelevant" alternatives. While this has been relaxed in many ways, most stochastic utility models place restrictions on the relative probabilities of choosing one option over another across distinct choice sets. For example, a weaker form asserts that, if one good is ever chosen with higher probability than an alternative in a given choice set, then it is chosen with higher probability than that alternative in all choice sets.

Our results show that there are weaker restrictions on standard stochastic choice data when imperfect perception is the source of stochasticity. The following example illustrates a complete 
reversal in choice probabilities between a two prize set and a three prize set. An item that is always chosen in two prize set is never chosen in the three prize set.

Example 4 Consider $X=\left\{x_{1}, x_{2}, x_{3}\right\}$ and assume that in binary choice with a symmetric layoutgenerating mechanism, $x_{1}$ is chosen with probability 1 over both $x_{2}$ and $x_{3}$, and $x_{2}$ is chosen with probability 1 over $x_{3}$,

$$
Q^{\left\{x_{1}, x_{2}\right\}}\left(x_{1}\right)=Q^{\left\{x_{1}, x_{3}\right\}}\left(x_{1}\right)=Q^{\left\{x_{2}, x_{3}\right\}}\left(x_{2}\right)=1 .
$$

This means that a necessary condition for RE-PREPs to exist for all three sets is that $U\left(x_{1}\right)>$ $U\left(x_{2}\right)>U\left(x_{3}\right)$.

$$
Q^{\left\{x_{1}, x_{2}\right\}}\left(x_{1}\right)=Q^{\left\{x_{1}, x_{3}\right\}}\left(x_{1}\right)=Q^{\left\{x_{2}, x_{3}\right\}}\left(x_{2}\right)=1 .
$$

The theorem implies that

$$
Q^{\left\{x_{1}, x_{2}, x_{3}\right\}}\left(x_{3}\right)=1
$$

is inconsistent with existence of a RE-PREP for the stochastic choice system. However, it is entirely possible to have $x_{2}$ chosen for sure,

$$
Q^{\left\{x_{1}, x_{2}, x_{3}\right\}}\left(x_{2}\right)=1
$$

In this case, one can construct a RE-PREP for the stochastic choice system in which the layoutgenerating mechanism is symmetric, the utility function satisfies $U\left(x_{1}\right)>U\left(x_{2}\right)>U\left(x_{3}\right)$, and prize $x_{2}$ is preferred to an equal chance of prizes $x_{1}$ and $x_{3}$,

$$
U\left(x_{2}\right)>\frac{U\left(x_{1}\right)+U\left(x_{3}\right)}{2} .
$$

The corresponding perception function is such that all prizes are perfectly identified in two prize sets, while there is complete inability to distinguish prizes 1 and 3 in three prize sets: the presence of prize $x_{2}$ induces confusion between $x_{1}$ and $x_{3}$.

While the above example is extreme, the broad idea is reasonable. Perceptual confusion may indeed be more significant in large choice sets than in small choice sets. As a result, a moderately good item that is easy to identify may be particularly attractive in large choice sets because there is little risk of it being confused with a bad item. Cases of this kind are modeled by Natenzon [2011], and fit well with the work of Iyengar and Lepper [2000] on difficulties associated with choice from large choice sets. 


\section{Experimental Evidence}

As indicated, a test of our theory requires rich data on the decision making "frame". We detail in this section a simple laboratory experiment in which: (1) the $\operatorname{ICE}(X, Y, \mu, P)$ is fully observed; and (2) the layout-generating mechanism $\mu$ is varied systematically. While subjects make substantial choice mistakes in all treatments, we find that the NIAS inequalities are always satisfied, so that subjects behave in accordance with our model.

Both of the framing effects allowed for in our model are present in the experimental data. Not only does the layout impact choice, but also the layout-generating mechanism. It is the latter effect that is most defining of our model, and the fact that we can control it experimentally is critical to our ongoing research on imperfect perception.

\subsection{Experimental Design}

In each round, subjects were presented with three options, each of which is composed of 20 numbers. Figure 4 shows a screen capture of a typical round. The value of each option is the sum of all 20 numbers, and subjects were incentivized to select the object with the highest value. In the baseline symmetric treatment ( $33 \%, 33 \%, 33 \%$ "), subjects were informed that all three options were equally likely to be the highest valued option, but in two other treatments, the first option was more likely to be the highest valued option. In one of the asymmetric treatments (" $40 \%, 30 \%, 30 \%$ "), subject were informed that the first option was approximately $40 \%$ likely to be the highest valued option (the other two were both approximately $30 \%$ likely). In the other asymmetric treatment (" $46 \%$, $27 \%, 27 \%$ "), subjects were told that the first option was approximately $46 \%$ likely to be the highest valued option (the other two were both approximately $27 \%$ likely). Subjects completed 12 rounds of each treatment, which were presented in a random order. ${ }^{4}$

\subsection{Testing for No Improving Action Switches (NIAS)}

The experiment was run with 36 subjects in the CESS laboratory. As seen in table 1, subjects chose the best (highest valued) option in $54 \%$ of rounds, which is higher than the expected percentage

\footnotetext{
${ }^{4}$ The instructions provided to subjects are available in the appendix.
} 


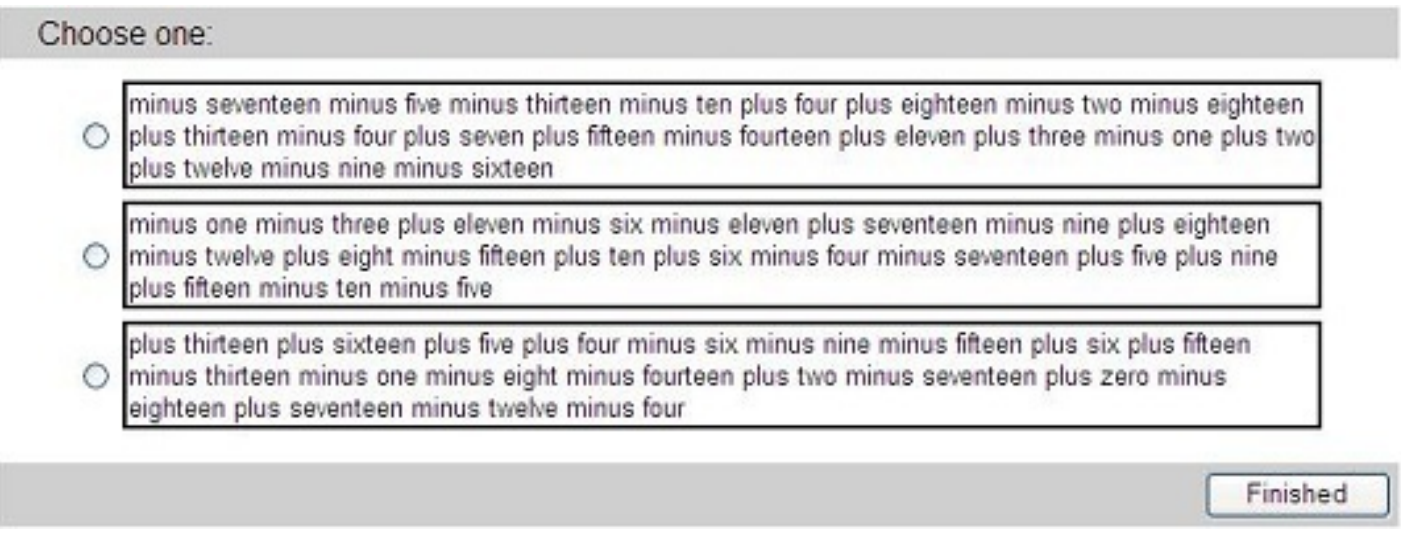

Figure 4: A typical round

under random choice but far below that associated with perfect perception. ${ }^{5}$ The fraction of rounds in which the highest valued option was chosen was stable over the course of the experiment - the fraction in the first half of rounds was not significantly different than the fraction in the second half of rounds $(\alpha=0.01)$.

Table 1. Percentage of rounds best option was chosen

\begin{tabular}{|l|l|l|}
\hline Treatment & $\mathrm{n}$ & Rounds best option chosen \\
\hline $33 \%, 33 \%, 33 \%$ & 418 & $54 \%$ \\
\hline $40 \%, 30 \%, 30 \%$ & 424 & $52 \%$ \\
\hline $46 \%, 27 \%, 27 \%$ & 422 & $56 \%$ \\
\hline Overall & 1264 & $54 \%$ \\
\hline
\end{tabular}

For a layout-generating mechanism $\mu$, the NIAS inequalities are satisfied for a utility function $U$ if,

$$
\sum_{n=1}^{N}\left[\delta_{m m}\left(x_{n}\right)-\delta_{m k}\left(x_{n}\right)\right] U\left(x_{n}\right) \geq 0,
$$

for every all $k, m \in\{1, \ldots, M\}$, with at least one inequality strict. If the utility function is normalized so that $U(\$ 8)=1$ and $U(\$ 4)=0$, then the NIAS inequalities reduce to,

$$
\frac{\sum_{\left\{f \in \mathcal{F} \mid f\left(y_{m}\right)=\$ 8\right\}} \mu(f) P^{f}\left(y_{m}\right)}{\sum_{f \in \mathcal{F}} \mu(f) P^{f}\left(y_{m}\right)}-\frac{\sum_{\left\{f \in \mathcal{F} \mid f\left(y_{k}\right)=\$ 8\right\}} \mu(f) P^{f}\left(y_{m}\right)}{\sum_{f \in \mathcal{F}} \mu(f) P^{f}\left(y_{m}\right)} \geq 0 .
$$

These conditions imply that the percentage of rounds in which action $y_{m}$ is chosen and is best must be no smaller, and sometimes strictly larger, than the percentage of rounds in which action

\footnotetext{
${ }^{5}$ More than one option had the highest value in $2.4 \%$ of rounds. These rounds were removed to simplify the analysis, but their exclusion does not qualitatively affect the results.
} 
$y_{m}$ is chosen, but action $y_{k}$ is best. This determines whether subjects could have done better by switching from action $y_{m}$ to action $y_{k}$ because the rounds where $y_{m}$ was chosen and was best would lose utility of 1 with the switch and the rounds where $y_{m}$ was chosen but $y_{k}$ was best would gain utility of 1 with the switch.

Table 2. Percentage of rounds chosen option was best by treatment

\begin{tabular}{|l|l|l|l|l|}
\hline & \multicolumn{4}{|l|}{ Best Option } \\
\hline Treatment & Chosen Option & 1st Option & 2nd Option & 3rd Option \\
\hline $33 \%, 33 \%, 33 \%$ & 1st Option & $48 \%$ & $25 \%$ & $27 \%$ \\
\hline & 2nd Option & $27 \%$ & $51 \%$ & $23 \%$ \\
\hline & 3rd Option & $19 \%$ & $24 \%$ & $56 \%$ \\
\hline $40 \%, 30 \%, 30 \%$ & 1st Option & $54 \%$ & $23 \%$ & $23 \%$ \\
\hline & 2 nd Option & $25 \%$ & $53 \%$ & $22 \%$ \\
\hline $46 \%, 27 \%, 27 \%$ & 1st Option & $54 \%$ & $19 \%$ & $27 \%$ \\
\hline & 2nd Option & $26 \%$ & $44 \%$ & $29 \%$ \\
\hline & 3rd Option & $17 \%$ & $23 \%$ & $60 \%$ \\
\hline
\end{tabular}

Table 2 shows, for the rounds in which a given action was taken, the percentage of rounds in which each option was the best option. This table can be used to directly verify that the NIAS inequalities hold for all treatments and all possible action switches. For example, in the " $40 \%, 30 \%$, $30 \%$ " treatment, the NIAS inequalities were satisfied when the first option was chosen because the percentage of rounds in which the first option was chosen and was best was $54 \%$, while the two alternative options were each optimal in only $23 \%$ of rounds in which the first option was chosen. In fact, the condition holds for all treatments and all possible action switches. The closest the NIAS condition comes to being violated is in the " $46 \%, 27 \%, 27 \%$ " treatment for switching from the second option (44\%) to the third option (29\%).

\subsection{Two Framing Effects}

As discussed previously, our model allows for two distinct forms of framing effects, and we see evidence of both in subjects' choices. As shown in table 3, we see slight evidence of order effects: the third option was selected less often than the second option, even though on average, there is 
no informational difference between the second and third option in any treatment. However, these proportions are only significantly different for the first treatment $(\alpha=0.05)$.

Table 3. Percent of rounds each option chosen by treatment

\begin{tabular}{|l|l|l|l|}
\hline Treatment & 1st Option & 2nd Option & 3rd Option \\
\hline $33 \%, 33 \%, 33 \%$ & $32 \%$ & $38 \%$ & $30 \%$ \\
\hline $40 \%, 30 \%, 30 \%$ & $57 \%$ & $23 \%$ & $20 \%$ \\
\hline $46 \%, 27 \%, 27 \%$ & $70 \%$ & $16 \%$ & $14 \%$ \\
\hline
\end{tabular}

Also present is the second framing effect, in which subjects respond to the information content of the layout-generating mechanism, in particular the probability with which each available action yields the best prize. The No Increasing Blind Switches (NIBS) condition requires that subjects choose the best option more often than they would if they "blindly" selected the first option. Table 1 above shows that this condition holds in every treatment because subjects did better on average than they would by always selecting the first option. In addition, the first column of table 3 shows that the percent of rounds in which the first option was chosen increases substantially as the likelihood of the first option being the best option increases and is significantly higher in the asymmetric treatments than the probability of the first option actually being the best option. These results raise intriguing questions concerning the interaction between the layout-generating mechanism and the quality of final choice.

\section{Concluding Remarks}

We have introduced a model of rational decision making that allows for an essentially unlimited array of perceptual limitations. We study "rational expectations perception-based representations" (RE-PREPs) in which DMs fully internalize how their perceptions relate to choices and to their consequences. We show that the signature of the resulting theory is that stochastic choices are "unimprovable", with the precise improvements that can be ruled out depending on the data available for model testing.

Our first characterization result involves a data set that includes the fine details of the decision making environment, in particular detailed information about how the decision was presented to the DM. In this context, we show that existence of a RE-PREP representation is equivalent to a 
No Improving Action Switches (NIAS) condition. We also characterize standard stochastic choice data consistent with our theory. The resulting restrictions, while intuitive, are weaker than those imposed by stochastic utility models.

Our model allows for both familiar framing effects that relate to the layout of the prizes and more novel effects that relate to the information content of the environment. We outline an experimental design to explore these effects, and first results support our model and indicate the importance of information-based framing effects. In ongoing work, we are further investigating the interaction between environmental information and imperfect perception, with particular focus on attention. We are also exploring the applicability of our general approach to questions of information processing and Bayesian inference, as well as to strategic settings in which agents may have different perceptions.

\section{Bibliography}

\section{References}

[1] Bernheim, B. Douglas, and Antonio Rangel. 2008. "Choice-Theoretic Foundations for Behavioral Welfare Economics." In The Foundations of Positive and Normative Economics, ed. Andrew Caplin and Andrew Schotter. New York: Oxford University Press.

[2] Block, H. D., and J. Marschak. 1960. "Random Orderings and Stochastic Theories of Response." In Contributions to Probability and Statistics. Essays in honor of Harold Hotelling, ed. I. Olkin. Stanford University Press.

[3] Caplin, Andrew. 2008. "Economic Theory and Psychological Data: Bridging the Divide." In The Foundations of Positive and Normative Economics, ed. Andrew Caplin and Andrew Schotter. New York: Oxford University Press.

[4] Caplin, Andrew, Mark Dean, and Daniel Martin. Forthcoming. "Search and Satisficing." American Economic Review.

[5] Caplin, Andrew, and Mark Dean. 2011. "Search, choice, and revealed preference." Theoretical Economics, 6: 19-48. 
[6] Compte, Olivier, and Andrew Postlewaite. 2011. "Mental Processes and Decision Making." Mimeo.

[7] Debreu, Gerard. 1960. "Review: Individual Choice Behavior." American Economic Review, 50(1): 186-188.

[8] Ergin, Haluk, and Todd Sarver. 2010. "A Unique Costly Contemplation Representation." Econometrica, 17: 1285-1339.

[9] Falmagne, J. C. 1978. "A representation theorem for finite random scale systems." Journal of Mathematical Psychology, 18(1): 52-72.

[10] Gabaix, Xavier. 2011. "A Sparsity-Based Model of Bounded Rationality.” Mimeo. NYU Stern.

[11] Geng, Sen . 2011. "Asymmetric search, asymmetric choice errors, and status quo bias." Mimeo. New York University.

[12] Gennaioli, Nicola, and Andrei Shleifer. 2010. "What Comes to Mind." Quarterly Journal of Economics, 125(4): 1399-1433.

[13] Glimcher, Paul. 2010. Foundations of Neuroeconomic Analysis, Oxford University Press.

[14] Gottlieb, Daniel. 2010. "Imperfect Memory and Choice Under Risk." Wharton Insurance and Risk Management Working Paper WP2010-05.

[15] Gul, Faruk, and Wolfgang Pesendorfer. 2008. "The Case for Mindless Economics." In The Foundations of Positive and Normative Economics, ed. Andrew Caplin and Andrew Schotter. New York: Oxford University Press.

[16] Gul, Faruk, and Wolfgang Pesendorfer. 2006. "Random Expected Utility." Econometrica, 74(1): 121-146.

[17] Iyengar, Sheena, and Mark Lepper. 2000. "When Choice is Demotivating: Can One Desire Too Much of a Good Thing?" Journal of Personality and Social Psychology, 79: 995-1006.

[18] Koszegi, Botond, and Matthew Rabin. 2008. "Revealed Mistakes and Revealed Preferences." In The Foundations of Positive and Normative Economics, ed. Andrew Caplin and Andrew Schotter. New York: Oxford University Press.

[19] Luce, R. Duncan. 1959. Individual Choice Behavior: a Theoretical Analysis. New York: Wiley. 
[20] McFadden, Daniel. 1973. Conditional logit analysis of qualitative choice behavior, Institute of Urban \& Regional Development, University of California.

[21] Manzini, Paola, and Marco Mariotti. 2007. "Sequentially Rationalizable Choice." American Economic Review, 97(5): 1824-1840.

[22] Masatlioglu, Yusufcan, and Daisuke Nakajima. 2009. "Choice by Iterative Search." University of Michigan Working Paper.

[23] Mullainathan, Sendhil. 2002. "A Memory-Based Model of Bounded Rationality." Quarterly Journal of Economics, 117: 735-774.

[24] Natenzon, Paulo. 2011. "Random Choice and Learning." Mimeo. Princeton University.

[25] Rabin, Matthew, and Georg Weizsacker. 2009. "Narrow Bracketing and Dominated Choices." American Economic Review, 99: 1508-1540.

[26] Ratcliff, Roger. 1978. "A theory of memory retrieval." Psychological Review, 85(2): 59-108.

[27] Ratcliff, Roger, and Gail McKoon. 2008. "The diffusion decision model: theory and data for two-choice decision tasks." Neural Computation, 20: 873-922.

[28] Reutskaja, Elena, Rosemarie Nagel, Colin Camerer, and Antonio Rangel. 2011. "Search dynamics in consumer choice under time pressure: An eyetracking study." American Economic Review, 101: 900-926.

[29] Rubinstein, Ariel, and Yuval Salant. 2006. "A Model of Choice from Lists." Theoretical Economics, 1(1): 3-17.

[30] Salant, Yuval, and Ariel Rubinstein. 2008. "("A", "f"): Choice with Frames," Review of Economic Studies, 75(4): 1287-1296.

[31] Schwartzstein, Joshua. 2010. "Selective Attention and Learning." Mimeo. Dartmouth College.

[32] Shadlen, Michael N., and William T. Newsome. 2001. "Neural basis of a perceptual decision in the parietal cortex (area LIP) of the rhesus monkey." Journal of Neurophysiology, 86: 19161936.

[33] Simon, Herbert. 1955. "A Behavioral Model of Rational Choice." Quarterly Journal of Economics, 69(1): 99-118. 
[34] Sims, Christopher A. 2003. "Implications of Rational Inattention." Journal of Monetary Economics, 50 (3), 665-690.

[35] Thaler, Richard H. 1985. "Mental Accounting and Consumer Choice." Marketing Science, 4, 199-214.

[36] Weber, E. H. 1834; 1996. On the Tactile Senses, ed. H. E. Ross and D .J.Murray. New York: Experimental Psychology Series.

[37] Wilson, Andrea. 2002. "Bounded Memory and Biases in Information Processing." Mimeo. Princeton University. 


\section{Appendix}

\section{Instructions}

In this experiment, you will take part in 1 practice round and then 36 regular rounds. Any choices you make during the practice round will not affect your final payment.

In each round, you will be shown 3 options, each of which contains 20 numbers. The value of each option is the sum of all 20 numbers. Your task will be to choose the option that has the highest value (or one of the options with the highest value). You cannot use a calculator or scratch paper.

At the end of the experiment, we will select 3 of the 36 regular rounds for payment. In each round selected for payment, if you chose the option with the highest value, you will get $\$ 8$, if not, you will get $\$ 4$. Thus, the maximum total payment is $\$ 24$ and the minimum total payment is $\$ 12$. When a round starts, you will be told which type of round it is. There are three different types of rounds:

1. "33\%, 33\%, 33\%" rounds (12 rounds total)

All options are equally likely (approximately 33\% likely) to have the highest value. For all options, each of the twenty numbers is a random integer between plus eighteen and minus eighteen (all equally likely), and each number is determined independent of the other numbers. Thus, the value of each option is independent of the value of the other options, and all options are equally likely to have the highest value.

2. " $40 \%, 30 \%, 30 \% "$ rounds ( 12 rounds total)

The first option is approximately $40 \%$ likely to have the highest value, and each of the others is approximately $30 \%$ likely to have the highest value. For the first option, each of the twenty numbers is a random integer between plus nineteen and minus eighteen (all equally likely). For the second and third option, each of the twenty numbers is a random integer between plus eighteen and minus eighteen (all equally likely). Once again, the value of each option is independent of the value of the other options.

3. "46\%, 27\%, 27\%" rounds (12 rounds total)

The first option is approximately $46 \%$ likely to have the highest value, and each of the others is approximately $27 \%$ likely to have the highest value. For the first option, each of the twenty numbers is a random integer between plus twenty and minus eighteen (all equally likely). For the second and third option, each of the twenty numbers is a random integer between plus eighteen and minus eighteen (all equally likely). Once again, the value of each option is independent of the value of the other options.

When a round starts, no option will be selected. You can change which option is selected by clicking on the button to the left of the option you want or by clicking anywhere on the option itself. You are free to change which option is selected at any time and as many times as you like.

Whenever you click on the 'Finished' button, the round will come to an end, and the selected option will be recorded as your choice. After a brief pause, you will be given the opportunity to either review the instructions again on the computer screen or proceed to the next round. This will continue until you have completed 1 practice rounds and 36 regular rounds, for a total of 37 rounds. 